\title{
Enfoque Ciencia Tecnología Sociedad y Ambiente CTSA como estrategia para el aprendizaje de la química en estudiantes de secundaria
}

\section{Approach Science Technology Society and Environment STSE as a strategy for learning chemistry in high school students}

DOI: http://dx.doi.org/10.17981/cultedusoc.11.2.2020.17

Recibido: 2 de febrero de 2020 Aceptado: 12 de mayo de 2020 Publicado: 12 de julio de 2020

\author{
María Cristina Murillo Durán \\ Instituto Técnico Agropecuario. Hato-Santander (Colombia) \\ mcristinamurillo@gmail.com
}

Elvira Tirado Santamaría

Universidad Autónoma de Bucaramanga. Bucaramanga (Colombia)

etirado@unab.edu.co

Para citar este artículo:

Murillo, M. y Tirado, E. (2020). Enfoque Ciencia Tecnología Sociedad y Ambiente CTSA como estrategia para el aprendizaje de la química en estudiantes de secundaria. Cultura, Educación y Sociedad, 11(2). 251-284. DOI: http://dx.doi.org/10.17981/ cultedusoc.11.2.2020.17

\section{Resumen}

En la actualidad en una sociedad dominada por la ciencia y la tecnología se requiere de ciudadanos científicamente formados, de allí la gran importancia de la ciencia que se imparte en las instituciones educativas. El objetivo principal del artículo es describir la investigación realizada en un ambiente rural en la que se desarrolla una secuencia didáctica bajo el enfoque Ciencia Tecnología Sociedad y Ambiente CTSA. Se utilizó un enfoque metodológico cualitativo de tipo investigación acción con la población estudiantil de una Institución oficial de carácter técnico ubicada en una población rural. Los resultados evidencian que los estudiantes se vieron más motivados por el aprendizaje de la química, más interesados por los conocimientos científicos y la relación con su entorno, así como un dominio conceptual de la temática seleccionada. Se puede concluir que este enfoque CTSA facilitó el aprendizaje significativo. Los estudiantes encontraron la química como una ciencia aplicable a su contexto y su futuro, construyendo una base para formar futuros ciudadanos interesados por el conocimiento científico.

Palabras clave: Secuencia didáctica; soluciones químicas; aprendizaje significativo; alfabetización científica

\begin{abstract}
At present, in a society dominated by science and technology requires citizens scientifically trained, hence the great importance of school science. The main objective of the article is to describe the investigation done in a rural environment in which a didactic sequence is developed under the Science Technology Society and Environment STSE approach. A qualitative methodological approach of action research type was used in the population of a Technical Agricultural Institute High School. The results show that the students were more motivated by the learning of Chemistry subject, more interested in scientific knowledge and the relationship with their environment, as well as a conceptual domain of the selected topic. It can be concluded that this STSE approach facilitated the meaningful learning. Students found in the Chemistry subject as a science applicable to their context and their future, building a basis for forming future citizens interested in scientific knowledge.
\end{abstract}

Keywords: Didactic sequence; chemical solutions; significant learning; scientific literacy 


\section{INTRODUCCIÓN}

Las diferentes problemáticas mundiales actuales tales como: el calentamiento global asociado a la acción del ser humano, la escasez de agua en muchas regiones del planeta, el mal manejo de las basuras, el abuso de los recursos naturales entre otros demuestra que se hace indispensable la formación de ciudadanos capaces de tomar decisiones científicas acordes a los retos afrontados por la sociedad actual, una sociedad permeada por la ciencia y la tecnología (Aikenhead, 2005).

Parte de esta formación se obtiene en la secundaria, específicamente en la asignatura de química, la química al ser la ciencia que estudia la materia y los cambios que en ella se dan, facilita el entendimiento de los fenómenos que rodean al ser humano y de las diferentes sustancias y materiales con las cuales nos encontramos a diario (Chang y College, 2002). Sin embargo, en la etapa escolar se presentan diferentes inconvenientes los cuales se ven reflejados en un bajo rendimiento de los estudiantes en esta materia y en una apatía duradera por la misma al percibirla abstracta, difícil y desligada de la realidad. Debido a esto los docentes de química se han preocupado por innovar en los procesos de enseñanzaaprendizaje, mejorando las prácticas educativas, encontrando nuevas formas para motivar al estudiante y lograr así un aprendizaje significativo en su asignatura.

El Colegio Instituto Técnico Agropecuario es la única institución educativa en el municipio del Hato Santander (Departamento de Santander, Colombia) municipio que se encuentra en las inmediaciones de la Serranía de los Yariguíes, también conocida como Serranía de los cobardes. Este municipio se encuentra a la altura de 1370 metros sobre el nivel del mar y tiene una temperatura promedio de $21^{\circ}$ centígrados, limita por el oriente con el municipio de El Palmar, al occidente con el municipio de El Carmen; al norte con el municipio de Galán y al sur con el municipio de Simacota. Su sede principal está ubicada en el sector rural, vereda "Centro" a solo 5 minutos de la cabecera municipal. En esta institución las asignaturas de ciencias naturales tienen un índice de aprobación bajo debido a que los estudiantes muestran dificultades significativas en competencias tales como planteamiento de problemas, uso de lenguaje científico y el uso del lenguaje matemático de modelos que explican fenómenos entre otras, además muchos estudiantes muestran desinterés por las ciencias naturales, situación que se repite en muchas instituciones educativas del departamento de Santander tal como lo evidencian estudios realizados en diferentes instituciones educativas, como los realizados por Botero y Palomeque (2014), Moreno (2016) y el realizado en el municipio vecino del Palmar por Mancilla (2017).

Según estos trabajos se espera que los alumnos logren aprendizajes de calidad con metodologías antiguas; se refleja la falta de métodos, estrategias y material adecuado para el desarrollo de las clases; se incorporan medios y nuevas tecnologías al proceso educativo, sin la debida práctica o supervisión y por ende no se obtienen resultados positivos.

Adicionalmente en las poblaciones rurales como la del Hato, se evidencian otras problemáticas que afectan a los estudiantes tales como las largas distancias recorridas por los mismos diariamente para llegar a clases, muchas veces caminando, además el mal estado de las carreteras le impide a los que viven en las veredas más alejadas asistir a clases en los días de lluvia. Algunos por sus bajos recursos económicos no ingieren las 3 comidas diarias y estudiantes que durante su tiempo libre apoyan la labor de sus padres en los 
trabajos del campo, situaciones que, muchas veces termina en indisposición hacia el colegio y la posible deserción, por esta razón con este trabajo se buscó emplear el enfoque CTSA con el fin acercar la ciencia a la cotidianidad del estudiante.

También se debe tener en cuenta la manera como muchos estudiantes perciben la ciencia y en particular la química, la ven como una asignatura solo comprensible para algunas personas, la asocian a conceptos complicados y abstractos difícilmente aplicables en su diario vivir. Moreira (2004) señala:

Enseñar ciencias tiene como objetivo: hacer que el alumno aprenda a compartir significados en el contexto de las ciencias, o sea, interpretar el mundo desde el punto de vista de las ciencias, manejar algunos conceptos, leyes y teorías científicas, abordar problemas razonando científicamente, identificar aspectos históricos, sociales y culturales de las ciencias (p. 01).

Se empleó el enfoque CTSA porque este busca humanizar la ciencia, haciéndola más accesible a todas las personas (Acevedo, Vázquez y Manassero, 2003), este enfoque surgió después de la segunda guerra mundial y se ha visto afectado por diferentes movimientos, sin embargo se considera al investigador Peter Fensham como la persona que lo consolidó en el área de la educación. Fensham (citado por Garritz, 2005) describió que en la actualidad el principal inconveniente del proceso de enseñanza aprendizaje en ciencias es la falta de interés de los estudiantes, y para abordarlo se requiere involucrar los aspectos actitudinales, afectivos y emocionales dentro del currículo escolar.

Se debe promover una actitud positiva en los estudiantes hacia la ciencia, suscitando la curiosidad y la motivación, de tal manera que se genere apego no solo a lo largo de la etapa escolar sino también de toda la vida. La propuesta de Fensham denominada ciencia para todos busca reducir la brecha entre la ciencia real y la impartida en las aulas de clase, para lograr así una alfabetización científica en la mayor parte de los ciudadanos (García y Cauich, 2008).

La problemática que aborda el enfoque seleccionado ha quedado constatada en diversos informes internacionales realizados por entidades internacionales como la Organización de las Naciones Unidas para la Educación, la Ciencia y la Cultura (UNESCO, 2003) el cual destaca que la educación en ciencias debe enfocarse en la necesidad de aplicar la ciencia y la tecnología en la resolución a problemas cotidianos, involucrando las diferentes teorías científicas, la ética y el control científico así como la interdependencia con las ciencias humanas.

Adicionalmente el gobierno colombiano a través de los estándares de competencias en ciencias naturales (Ministerio de Educación Nacional, MEN, 2006) se ha unido a los esfuerzos por implementar este enfoque en las aulas de clase, ya que en ellos para todos los bloques de grados se especifica un grupo de competencias relacionadas con la interacción ciencia, tecnología y sociedad.

Teniendo en cuenta la problemática anteriormente descrita se planteó la siguiente pregunta de investigación: ¿Cómo lograr un aprendizaje significativo de química en estudiantes de secundaria del Instituto Técnico Agropecuario del municipio de Hato Santander utilizando el enfoque Ciencia Tecnología Sociedad y Ambiente CTSA?

Para responder a esta pregunta en la investigación realizada se planteó como objetivo general: favorecer el aprendizaje significativo de la química en los estudiantes de noveno grado del Instituto Técnico Agropecuario de Hato-Santander empleando elementos propios 
de su realidad socioeconómica-cultural con la implementación de una secuencia didáctica bajo el enfoque CTSA. Los objetivos específicos obedecieron a cada una de las etapas de la investigación, estos iban desde identificar los elementos sociales, económicos y culturales a los cuales están expuestos los estudiantes y que pueden ser empleados para el desarrollo de las clases mediante diferentes recursos, hasta evaluar las competencias alcanzadas por los estudiantes en la asignatura de química específicamente en el análisis de soluciones y las relaciones cuantitativas soluto-solvente.

La investigación fue realizada en el Instituto Técnico Agropecuario, única institución educativa en el municipio de Hato Santander. El municipio cuenta 9 veredas con diversidad de pisos térmicos, por ello la economía del Hato es eminentemente agropecuaria. Según el Plan de Ordenamiento Territorial del municipio-POT (Alcaldía Municipal Hato, 2016) los renglones económicos más sobresalientes son: la caña panelera ya que en la actualidad existen aproximadamente 42 trapiches entre tecnificados y manuales, el café debido a que el 80\% familias hateñas son cultivadoras y un tercer renglón significativo en la economía lo constituye la actividad pecuaria y de una manera muy especial la ganadería vacuna, aunque también de especies menores como caprinos, porcinos, peces y aves.

La institución busca brindar una educación que responda a las condiciones de vida rural, a sus expectativas, necesidades y posibilidades. Por esta razón cuenta con una especialidad técnica agropecuaria y actualmente con un convenio con el Servicio Nacional de Aprendizaje SENA.

\section{Constructivismo y aprendizaje significativo}

En esta investigación se buscó lograr un aprendizaje significativo mediante una secuencia didáctica, entonces ¿qué es el aprendizaje significativo? Es un término proveniente de la teoría propuesta por Ausubel, Novak y Hanesian (1983) en la cual se entregan los mecanismos capaces de llevar a la adquisición y retención de los cuerpos de significados manejados en las aulas, es decir, en lo que el alumno aprende. Aborda las situaciones de ese aprendizaje, las condiciones en las cuales se produce y los resultados obtenidos. La teoría de Ausubel involucra los elementos, factores, condiciones que se deben tener en cuenta si se busca garantizar que el estudiante pueda dar significado a los contenidos vistos en la etapa escolar (Silva, 2011).

El conocimiento previo es, en la visión de Ausubel, la variable más importante para el aprendizaje significativo de nuevos conocimientos. Aquello que ya se encuentra en la estructura cognitiva del sujeto que aprende puede facilitar el aprendizaje significativo, sin embargo, eso no significa que sea siempre una variable facilitadora. Normalmente sí, pero, en algunos casos, puede ser bloqueadora (Díaz y Hernández, 2002; Moreira, Caballero y Rodríguez, 1997).

Se tomaron en cuenta los principios constructivistas, provenientes de una corriente que generalmente se le atribuye a Jean Piaget quien sugirió que, los individuos construyen nuevos conocimientos a partir de las experiencias vividas, a través de procesos de acomodación y asimilación. La asimilación es posible únicamente cuando el nuevo conocimiento se alinea con la representación interna del mundo (Díaz y Hernández, 2002; Romero, 2009). 
En esta corriente se asume que en la escuela los alumnos aprenden y se desarrollan en cuanto logran construir significados adecuados en torno a los contenidos pertenecientes a el currículum escolar. Al estudiante se le considera el centro del proceso de enseñanza, y el objetivo de esta es potenciar sus capacidades. El aprendizaje no limita su incidencia a las capacidades cognitivas, en su lugar afecta a todas las capacidades y repercute en el desarrollo global del alumno (Romero, 2009).

En el constructivismo se busca poner al estudiante como el centro del proceso educativo construyendo su propio conocimiento, pero: ¿Es aplicable esto a las ciencias naturales? ¿Es realmente posible que el estudiante construya el conocimiento científico cuando este llevó siglos para llegar estado actual? Autores como Gil, Carrascosa y Martínez (1999) y Pozo (1987), señalan que los estudiantes no pueden construir todos los conocimientos científicos porque muchos de los conceptos centrales de la ciencia son bastantes difíciles de descubrir para la mayor parte de los adolescentes e incluso de los universitarios, por lo cual es difícil aplicar una propuesta totalmente constructivista a las ciencias naturales, sin embargo, hay muchos trabajos donde se aborda esta metodología, como los de Moreira (2004) quien señala: se debe diferenciar la educación en ciencias del entrenamiento científico, diferenciar construir conocimiento con "hacer ciencia" (p. 2). Buscar que el estudiante construya su propio conocimiento en ciencias no implica poner al alumno constantemente en el laboratorio y pretender que descubra todo por sí mismo, sino más bien guiarlo para que pueda abordar los problemas y situaciones científicamente.

Como se mencionó anteriormente con esta investigación se buscó un primer paso para hacer de los estudiantes del ITA ciudadanos científicamente alfabetizados, este es también el objetivo del movimiento ciencia para todos impulsado por Fensham en la década de los 80 y en el cual se basa el enfoque de este trabajo, se refiere a la necesidad de formar ciudadanos científicamente alfabetizados pero esta alfabetización científica se puede entender de diferentes maneras:

García \& Cauich (2008) describen la alfabetización científica como educar para conocer mejor las teorías científicas, la historia de las disciplinas, la ética y el control científico, la naturaleza del trabajo científico y la interdependencia entre ciencia, tecnología, sociedad y humanidades, además de formar para entender cómo se aplican la ciencia y la tecnología en la resolución de problemas cotidianos (p. 115). Reiss, Millar y Osborne (1999) la describieron como la formación para aprender, durante toda la vida, conceptos, habilidades y actitudes para ser científico, ingeniero o técnico. $\mathrm{O}$ sea, para comprender cómo se genera y prueba el conocimiento, cómo investigar, cómo extraer conclusiones desde la evidencia, como resolver problemas y tomar decisiones.

Para Furió, Vilches, Guisasola y Romo (1997) citados en Sabariego y Manzanares (2006), la alfabetización científica es la formación para que la población disponga de los conocimientos científicos y tecnológicos necesarios para desenvolverse en la vida diaria, ayudar a resolver los problemas y necesidades de salud y supervivencia básicos, tomar conciencia de las complejas relaciones entre ciencia y sociedad y, en definitiva, considerar la ciencia como parte de la cultura de nuestro tiempo.

Este trabajo, así como muchos otros en la actualidad promueve como finalidad central de la enseñanza de las ciencias la alfabetización científica y tecnológica de la ciudadanía 
por las razones aquí expuestas; otros autores suelen justificarlo apelando a la importancia de la participación ciudadana en la toma de decisiones directamente relacionadas con la ciencia y la tecnología así como a las repercusiones que tendrán estas sobre su calidad de vida, también apelan motivos culturales, de autonomía personal y cívicos (Acevedo et al., 2003).

A continuación, se definirán algunos conceptos que tuvieron importancia a lo largo de la investigación ya que están relacionados con las categorías de análisis seleccionadas y con la metodología empleada:

Motivación: Cuando se describió la teoría del aprendizaje significativo se vio que uno de los factores más importantes para lograrlo es la motivación del estudiante hacia el aprendizaje y aumentar la motivación es también una de las metas del enfoque CTSA.

Çınar, Bektaş y Aslan (2011) describen el término motivación acudiendo a su origen en la palabra latina "moveré" cuyo significado es mover (p. 87). La motivación representa el proceso que despierta, activa, dirige y sostiene el comportamiento y el rendimiento. Puede verse también como el proceso de estimulación de las personas a la acción para lograr una tarea deseada. Una persona está motivada cuando quiere hacer algo.

Ryan \& Deci (2000) describen dos tipos de motivación: la intrínseca y la extrínseca, la motivación intrínseca está relacionada con la curiosidad, la búsqueda de elementos novedosos, el disfrute de los retos, el crecimiento personal, entre otros, mientras, la motivación extrínseca se refiere a aquella que busca un resultado externo, puede ser un premio, reconocimiento, aceptación de un grupo.

Concepciones alternativas o ideas previas: Aunque este trabajo no corresponde a un estudio sobre las concepciones alternativas o ideas previas de los estudiantes, se consideró necesario tenerlas presentes a lo largo de la investigación, ya que, como se mencionó en la teoría del aprendizaje significativo de Ausubel la mente de los estudiantes posee una estructuración conceptual, donde los mismos le han dado explicación a las experiencias vividas, generando teorías personales relacionadas con lo que se quiere enseñar, por lo tanto resulta de vital importancia conocer previamente qué sabe el estudiante antes de pretender enseñarle algo (Oliva, 1999).

Castillo, Ramírez y González (2013) definen las concepciones alternativas o ideas previas como aquellas construidas por los sujetos donde elaboran respuestas a su necesidad de interpretar fenómenos naturales, aun cuando estas construcciones no obedezcan a los constructos aceptados por la ciencia, generalmente no corresponden un modelo o representación muy coherente y estable.

Conflicto cognitivo: Es un término empleado por Piaget para referirse al cambio conceptual generado en los estudiantes cuando existe una situación contradictoria entre lo que ellos saben (concepciones alternativas) y los nuevos conocimientos lo cual genera un desequilibrio cognitivo que conduce a un nuevo conocimiento más amplio y ajustado a la realidad.

Moreira y Greca (2003) señalan tener en cuenta el conflicto cognitivo es de vital importancia en el proceso de enseñanza aprendizaje, aunque no siempre resulta suficiente para rechazar definitivamente una concepción alternativa ya que los estudiantes en ocasiones proponen hipótesis auxiliares que logran salvar sus teorías implícitas previas, por esta razón el docente debe estar atento procurando un verdadero cambio conceptual. 
Secuencia didáctica: La intervención realizada con los estudiantes en esta investigación fue una secuencia didáctica, la cual, Díaz-Barriga (2013) define como aquella secuencia constituida por una serie de actividades de aprendizaje organizadas para permitir a los estudiantes crear situaciones donde se facilite obtener un aprendizaje significativo, estas actividades deben tener un orden interno y deben encontrarse relacionadas entre sí, el docente busca recuperar las nociones que tienen los estudiantes sobre un fenómeno o hecho y las vincula con situaciones problemáticas relacionadas con el contexto procurado que la información a la que va a acceder el estudiante sea significativa, es decir, tenga sentido y pueda facilitar el proceso de aprendizaje (DíazBarriga, 2013; Otero, 2014).

\section{MetodoloGía}

La pregunta de investigación en la que se basó la investigación es ¿Cómo lograr un aprendizaje significativo de química en estudiantes de secundaria del Instituto Técnico Agropecuario del municipio de Hato Santander utilizando el enfoque Ciencia Tecnología Sociedad y Ambiente CTSA? Para responderla se eligió un enfoque metodológico netamente cualitativo, teniendo en cuenta que según lo planteado en los objetivos no solo se desea saber si hubo aprendizaje de parte de los estudiantes, si no también abordar de qué manera ven ellos su entorno, cómo analizan la relación entre la temática tratada en clase y la realidad socio-cultural que ellos viven, para poder conocer si al utilizar el enfoque CTSA se obtuvieron los resultados propuestos.

El tipo de estudio empleado es el de investigación acción, ya que autores como Hernández, Fernández y Baptista (2014), Miguélez (2000), Ñaupas, Valdivia, Palacios y Romero (2016) señalan que este tipo de estudio tiene como finalidad comprender y resolver problemáticas específicas de una colectividad, en este caso una población netamente rural que tiene unas condiciones socio-económicas particulares.

Sandín (2003) define el proceso de investigación-acción como una espiral de cambio donde se pueden diferenciar al menos cuatro fases las cuales estuvieron presentes en esta investigación: la planificación, la acción, la observación y la reflexión, sin embargo, señalando que el proceso es dinámico e interactivo por lo cual frecuentemente debido a la reflexión existen cambios entre ellas.

\section{Diseño}

Para este trabajo se establecieron unas categorías iniciales como motivación hacia el aprendizaje, efecto del enfoque CTSA y dominio conceptual de la temática tratada las cuales fueron observadas a lo largo de todo el proceso, sin embargo, se estuvo atento al posible surgimiento de categorías emergentes que ayudaran a entender el fenómeno estudiado y si el enfoque CTSA facilitó el aprendizaje significativo en los estudiantes, durante la investigación se consideró incluir las concepciones alternativas como categoría emergente, sin embargo, se descartó debido a que hacía este estudio mucho más extenso y podrían no abordarse con la rigurosidad deseada. Sería recomendable en una investigación posterior tenerla en cuenta como categoría inicial. 
Una de las técnicas más efectivas para el presente estudio fue la observación participante, por lo cual en todas las secciones se usó el diario de campo donde se registró cada evento observado y las afirmaciones empleadas por los estudiantes, también se tuvo en cuenta el cuestionario y un test; los cuales permitieron recoger los insumos para los respectivos análisis, reflexiones y futuras planeaciones de actividades. Para identificar los elementos sociales, económicos y culturales a los que están expuestos los estudiantes se realizó un análisis documental recurriendo a los archivos municipales y de la institución, también se les aplicó un cuestionario a los estudiantes involucrados con el fin de reafirmar la información recolectada previamente e identificar factores posibles factores diferentes a los anteriores.

La investigación realizada contó con tres fases las cuales se plantearon con el fin de alcanzar los objetivos del proyecto. La primera fase correspondió a la revisión documental para analizar el contexto de la institución, la cual fue reforzada por un cuestionario de caracterización que se aplicó a los estudiantes que conformaron la muestra para identificar elementos que pudieron quedar fuera durante la primera fase, durante esta fase también se elaboró un perfil motivacional empleando un instrumento adaptado del desarrollado por Tuan, Chin \& Shieh (2005), que permitió conocer los intereses motivacionales de los estudiantes y así orientar la secuencia didáctica empleando el enfoque escogido.

La segunda fase corresponde a la implementación de la secuencia didáctica diseñada, esta constó de 6 secciones las cuales fueron aplicadas a lo largo de mes y medio, la primera sección estaba dedicada a la exploración de preconceptos relacionados con la composición de la materia y a introducir brevemente la temática de las soluciones químicas mostrando algunas situaciones donde se aplican, en la segunda sección se buscaba que los estudiantes mediante la observación de elementos de su cotidianidad, lograran diferenciar y clasificar la materia de acuerdo a la presencia de diferentes fases en ella. La tercera sección permitió a los estudiantes identificar las condiciones que afectan la solubilidad de una sustancia en un solvente y se facilitó la clasificación de las soluciones de acuerdo con la relación soluto/ solvente. La sección 4 tenía como finalidad otorgar a los estudiantes situaciones que les permitieran identificar algunas de las soluciones químicas en su contexto y finalmente las secciones 5 y 6 explicar a los estudiantes las diferentes relaciones cuantitativas entre el soluto y el solvente.

En último lugar se realizó la fase de evaluación, en ella mediante una prueba escrita se buscó conocer el alcance de logro alcanzado por los estudiantes referente a la temática de soluciones químicas, esto ayudó a valorar el impacto que generó la puesta en marcha del proyecto en los estudiantes del grado noveno quienes integraron la muestra del proyecto.

\section{Población y muestra}

La investigación se dirigió a los estudiantes de básica secundaria del Instituto Técnico Agropecuario del municipio de Hato Santander, 187 estudiantes hombres y mujeres en un rango de edades entre los 11 y los 19 años provenientes mayoritariamente de las 9 veredas del municipio.

El grupo seleccionado correspondió a los 27 estudiantes que hacían parte del grado noveno del Instituto Técnico Agropecuario, adolescentes entre los 14 y 16 años, se escogió 
este grupo debido a que es en grado noveno cuando los estudiantes tienen acceso por primera vez a la asignatura de química dentro de la institución y donde se ha identificado mayor apatía de parte de los estudiantes hacia la asignatura.

\section{Técnicas e instrumentos de recolección de datos}

Una de las técnicas más efectivas para esta investigación fue la observación participante, pero también se tuvo en cuenta el cuestionario y una prueba escrita; las cuales permitieron recoger los insumos para los respectivos análisis, reflexiones y futuras planeaciones de actividades. Para identificar los factores sociales, económicos y culturales a los que están expuestos los estudiantes se realizó un análisis documental recurriendo a los archivos municipales y de la institución, también se aplicó un cuestionario a los estudiantes involucrados con el fin de reafirmar la información con la que se contaba e identificar factores que no pudieron encontrarse en la revisión documental o análisis, hay que señalar que este no buscaba hacer un análisis cuantitativo de los estudiantes, si no más bien, recolectar información sobre sus pensamientos, actitudes y motivaciones hacia la asignatura mediante preguntas en su mayoría abiertas, también se empleó para recolectar alguna información sobre su forma de vida y la de sus familias. Todos los instrumentos empleados durante la investigación fueron validados previamente por expertos.

\section{ANÁLisis DE RESULTAdos}

Para poder analizar los resultados obtenidos inicialmente se examinaron los obtenidos en el cuestionario de caracterización y luego se realizó un análisis de los resultados a lo largo de la secuencia didáctica para cada una de las categorías propuestas.

\section{Resultados cuestionario de caracterización}

Con el fin de conocer y determinar los factores sociales, económicos y culturales a los que están expuestos los estudiantes se aplicó un cuestionario donde se indagó a los estudiantes sobre su vida cotidiana. Este permitió conocer que de los 27 estudiantes 24 son nacidos en la región, tres de ellos son nacidos en otras ciudades del país, aunque llevan la mayor parte de su vida en el municipio del Hato por lo que se encuentran profundamente arraigados a la región, a la cultura y costumbres de esta.

Se evidenció que la mayor parte de los estudiantes depende económicamente de la agricultura y de labores relacionadas con el campo, un 10\% manifiesta tener alguna microempresa como una carnicería o carpintería, también hay dos estudiantes que dependen económicamente de la labor de sus madres en oficios generales.

Se les indagó sobre si en algún momento del año realizan algún tipo de actividad para apoyar económicamente a su familia, y la mitad de los estudiantes manifestó trabajar en labores del campo con tal fin, especialmente durante la temporada de cosecha de café y de caña de azúcar. Los 27 estudiantes aseguraron que apoyan en las labores cotidianas del hogar. 


\section{Categoría de análisis: motivación}

Para realizar el análisis de la categoría motivación cada estudiante respondió al instrumento Test orientaciones motivacionales hacia el aprendizaje de la química adaptado del cuestionario desarrollado por Tuan et al. (2005), constituido por 35 afirmaciones, en las orientaciones motivacionales: autoeficacia, estrategias para aprender, percepción de la importancia de la química, logro de la meta, y entorno de aprendizaje, se tomó el promedio de las afirmaciones para cada orientación y se graficó en un diagrama radial, de esta manera se pudo observar de manera gráfica las orientaciones motivacionales de cada estudiante y del promedio del grupo, este procedimiento se realizó antes de implementar la secuencia didáctica para tener en cuenta esta información en la aplicación y diseño.

A continuación, se muestra la gráfica de un estudiante, el objetivo con este instrumento y la Figura 1 que surgió del mismo no fue realizar un análisis cuantitativo, si no conocer las tendencias motivacionales de cada estudiante para tenerlas en cuenta al elaborar la secuencia didáctica y analizar mejor los resultados.

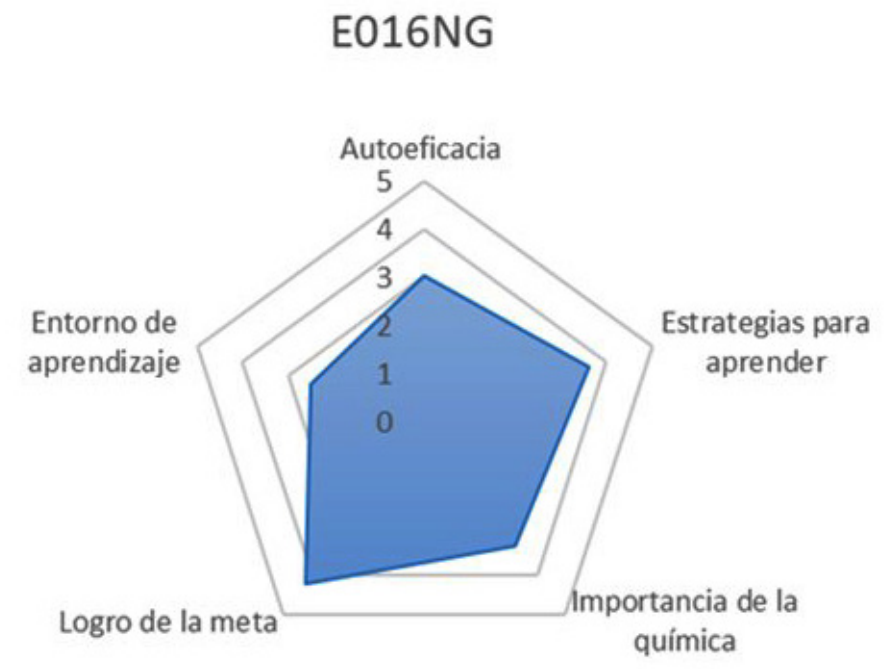

Figura 1. Perfil motivacional estudiante E016NG

Fuente: Basado en el cuestionario desarrollado por Tuan et al. (2005).

Como lo menciona Lobo (2016), los estudiantes están motivados por una combinación de diferentes factores. Para tener una idea de cuáles son esos factores se realizaron algunas preguntas concretas que hacían referencia a que tan motivados y dispuestos estaban a participar en la clase de química, estas fueron ¿Qué lo motiva a estar en clase de química? Seguido de ¿le gusta la clase de química? Se encontró que una parte considerable del grupo manifiesta que le gusta la clase a veces, dependiendo de la metodología empleada, el tema visto, bien sea por que lo consideren interesante o fácil, sus respuestas se contrastaron con el perfil motivacional encontrando que eran congruentes con el mismo.

Después se analizó la categoría motivación por el aprendizaje en los estudiantes a través de las secciones que comprendieron la secuencia didáctica, se destaca que en la exploración de preconceptos se les explicó que esta prueba tendría un carácter diagnóstico por lo que no tendría repercusión en su desempeño académico, por tal razón dos estudiantes manifestaron no desear responder la prueba si no afectaba sus notas evidenciándose de esta manera una motivación bastante extrínseca. 
En la segunda sección se mostraron más motivados por la novedad, como menciona Naranjo (2009) las novedades suelen ser más atractivas y motivadoras que aquello ya conocido. En la tercera sección la seguridad que dio la experiencia previa realizada hizo que los estudiantes se sintieran más capaces, motivándolos a continuar. En las secciones 5 y 6 se vieron dificultades en los procesos numéricos lo cual terminó en desmotivación de los estudiantes.

\section{Categoría de análisis: efecto del enfoque CTSA}

Se consideró que antes de cuestionar si hubo o no un aprendizaje significativo es pertinente conocer el enfoque seleccionado tuvo algún efecto en los estudiantes.

Como punto de partida en el cuestionario de caracterización se incluyó la pregunta ¿Considera que lo visto en clase de química puede llegar a ser aplicado en algún elemento de su contexto? ¿en cuál? Menos de la mitad de los estudiantes respondió que Si, la mayoría de los que respondieron afirmativamente mencionaron ejemplos únicamente académicos.

En las primeras secciones se observó sorpresa por el uso de elementos cotidianos y comparación con situaciones cotidianas realizadas por los mismos estudiantes. En las secciones posteriores se observó que los estudiantes comenzaron a manifestar en su discurso la aplicabilidad de la química, así como mayor propiedad al hablar de ciencias. Al finalizar la secuencia didáctica se realizó la misma pregunta con la que se inició el proceso, la mayoría respondió que sí y emplearon argumentos más sólidos que los empleados inicialmente.

\section{Categoría de análisis: dominio conceptual}

Al finalizar la secuencia didáctica se trató de identificar si los estudiantes alcanzaron diferentes niveles de conocimiento relacionado con las soluciones, para realizar el análisis se establecieron los niveles: reconocimiento, comprensión y cuantificación. Se pudo evidenciar que los estudiantes alcanzaron el nivel de comprensión, es decir lograron reconocer una solución, identificarlas en su vida cotidiana entender que estos conocimientos pueden llegar a ayudarles a solucionar problemas en su vida cotidiana. Del grupo de estudiantes solo 7 alcanzaron el nivel de cuantificación es decir lograron expresar en lenguaje matemático la relación soluto solvente y fueron capaces de calcular la concentración de una solución en los diferentes términos físicos. Del grupo únicamente una no alcanzó ni siquiera el nivel de reconocimiento ya que mostró dificultades a pesar de sus esfuerzos por lograrlo.

\section{Conclusiones}

En el desarrollo de la investigación realizada con miras al aprendizaje significativo de la química empleando el enfoque CTSA, surgen una serie de conclusiones referentes tanto al avance de cada una de las etapas como a los resultados observados después de implementar la secuencia didáctica. 
- La revisión documental y la caracterización aplicada al inicio de la investigación, así como el test aplicado con miras a establecer la motivación que tienen los estudiantes hacia la asignatura de química, brindó un insumo para el diseño y posterior implementación de la secuencia didáctica. Esto permitió que la investigadora brindara especial atención al desarrollo de prácticas que permitieran elevar el factor motivacional, comprender algunas actitudes de los estudiantes y a partir de esto tratar de imprimir mayor dinamismo y aumentar el nivel de dificultad de las actividades de forma gradual y cuidadosa, tratando así de bajar la percepción de dificultad arraigada en ellos.

- Se diseñó una secuencia didáctica empleando los factores sociales económicos y culturales identificados, esta estaba dividida en una exploración de preconceptos y 5 secciones adicionales, cada una estaba pensada para trabajar un objetivo diferente, directamente relacionada con la temática elegida.

- Para la etapa de implementación de la secuencia didáctica, se pueden mencionar entre los factores más importantes el motivacional debido a que en los estudiantes juega un papel fundamental en su proceso de aprendizaje significativo, es así como el simple hecho de cambiar su lugar habitual de trabajo durante las primeras secciones eleva su interés y atención notablemente, este interés aumenta aún más si se usan elementos que pertenecen a su realidad y que no les resultan ajenos ni abstractos, sin embargo en una pequeña cantidad de estudiantes el uso de estos elementos limitó el componente novedoso que ellos percibían de la actividad, bajando así su interés.

- La exploración de preconceptos permitió establecer las fortalezas y debilidades de los estudiantes, así como algunas concepciones alternativas que se aplican a los conceptos de química. A partir de estos resultados, se pudieron establecer cuales temas requerían mayor dedicación y en cuales se podía trabajar con una rigurosidad menor.

- A través de la secuencia didáctica, se facilitaron los procesos de comprensión, análisis y aplicación de las soluciones químicas, debido a que los estudiantes manifestaron que encontraban lo tratado en clase un poco más sencillo y comprensible, así como se observó en ellos una mejor percepción referente a la utilidad de la asignatura, dejándola de percibir deshumanizada y abstracta.

Para terminar y dando respuesta a la pregunta ¿Cómo lograr un aprendizaje significativo de química en estudiantes de secundaria del Instituto Técnico Agropecuario del municipio de Hato Santander utilizando el enfoque Ciencia Tecnología Sociedad y Ambiente CTSA? Se encontró que emplear una secuencia didáctica con este enfoque facilitó una mayor motivación por el aprendizaje, aumentando el interés y facilitando así el aprendizaje significativo de los estudiantes, tal como se puede evidenciar en los resultados positivos observados a lo largo de las diferentes secciones y en la prueba final aplicada. Emplear los factores sociales, económicos y culturales de la región permitió además que los estudiantes encontraran la química como una ciencia más real, aplicable a su contexto y su futuro, viéndola así menos abstracta y construyendo así una base para formar futuros ciudadanos interesados por el conocimiento científico y capaces de tomar decisiones en un mundo rodeado de este tipo conocimiento. 


\section{REFERENCIAS}

Acevedo, J. A., Vázquez, Á. y Manassero, M. A. (2003). Papel de la educación CTS en una alfabetización científica y tecnológica para todas las personas. Revista electrónica de Enseñanza de las Ciencias, 2(2), 80-111. Recuperado de http://reec.uvigo.es/volumenes/volumen2/REEC_2_2_1.pdf

Aikenhead, G. (2005). Educación Ciencia-Tecnología-Sociedad (CTS): una buena idea como quiera que se le llame. Educación Química, 16(2), 114-124. https://doi.org/10.22201/ fq.18708404e.2005.2.66121

Alcaldía Municipal Hato. (2016). Plan de desarrollo 2016 - 2019 Municipio de Hato - Santander. [Online]. Recuperado de https://hatosantander.micolombiadigital.gov.co/sites/ hatosantander/content/files/000023/1128_plan-de-desarrollo-2016-2019-web.pdf

Ausubel, D., Novak, J. y Hanesian, H. (1983). Psicología Educativa: Un punto de vista cognoscitivo. (2 Ed.) México, D.D.: Trillas.

Botero, J. F. y Palomeque, L. A. (junio, 2014). El OVA como Estrategia para la Enseñanza Aprendizaje de la Cinética Química. Presentado a IX Conferencia Latinoamericana de Objetos yTecnologías de Aprendizaje, Laclo 2014, (pp. 737-740). Manizales, Colombia. Recuperado de http://www.dnia.unal.edu.co/sites/default/files/documentos/306-1168-1PB.pdf

Castillo, A., Ramírez, M. y González, M. (2013). El aprendizaje significativo de la química: condiciones para lograrlo. Omnia, 19(2), 11-24. Disponible en https://produccioncientificaluz.org/index.php/omnia/article/view/7399

Chang, R. y College, W. (2002). Química. (7 ed.). México, D.F.: McGraw-Hill.

Çınar, O., Bektaş, Ç. y Aslan, I. (2011). A motivation study on the effectiveness of intrinsic and extrinsic factors. Economics and management, 16, 690-695. Disponible en http:// ecoman.ktu.lt/index.php/Ekv/issue/archive

Díaz-Barriga, Á. (2013). Guía para la elaboración de una secuencia didactica. México, D.F.: UNAM. Recuperado de http://www.setse.org.mx/ReformaEducativa/Rumbo\%20 a\%20la\%20Primera\%20Evaluaci\%C3\%B3n/Factores\%20de\%20Evaluaci\%C3\%B3n/ Pr\%C3\%A1ctica\%20Profesional/Gu\%C3\%ADa-secuencias-didacticas_Angel\%20 D\%C3\%ADaz.pdf

Díaz, F. y Hernández, G. (2002). Estrategias docentes para un aprendizaje significativo. (2 ed.). México, D.F.: Mc Graw Hill.

Furió, C., Vilches, A., Guisasola, J. y Romo, V. (1997). Finalidades de la Enseñanza de las Ciencias en la secundaria obligatoria; ¿Alfabetización científica o preparación propedéutica? Enseñanza de las Ciencias, 19(3), 365-376. Recuperado de https://core.ac.uk/ download/pdf/38990675.pdf

García, J. \& Cauich, J. (2008). ¿Para qué enseñar ciencias en la actualidad? Una propuesta que articula la tecnología, la sociedad y el medio ambiente. Revista Educación y Pedagogía, 50(1), 111-122. Recuperado de https://revistas.udea.edu.co/index.php/revistaeyp/article/view/9929

Garritz, A. G. (2005). Ciencia para todos. Un proyecto que dura ya 20 años. Educación Química, 16(1), 2-7 Recuperado de http://www.revistas.unam.mx/index.php/req/article/download/66129/58041 
Gil, D., Carrascosa, J. y Martínez, F. (1999). El surgimiento de la didáctica de las ciencias como campo específico de conocimientos. Revista Educación y Pedagogía, 11(25), 1365. Disponible en https://revistas.udea.edu.co/index.php/revistaeyp/article/view/5859

Hernández, R., Fernández, C. y Baptista, P. (2014). Metodología de la investigación. Mexico, D.F.: McGraw-Hill Education.

Lobo, R. V. (2016). Desarrollo de la motivación a través de la implementación de situraciones problema sobre la densidad. Actualidades Pedagógicas, (70), 51-73. https://doi. org/10.19052/ap.4093

Mancilla, A. R. (2017). Diseño de una guía de aprendizaje sobre estequiometria utilizando la herramienta drive para estudiantes de $10^{\circ}$ grado del municipio Palmar Santander. [Trabajo grado]. Universidad Francisco de Paula Santander, Palmar, Colombia. Recuperado de http://repositorio.ufpso.edu.co:8080/dspaceufpso/handle/123456789/1908

Miguélez, M. (2000). La investigación-acción en el aula. Agenda académica, 7(1), 27-39. Recoperado de http://files.docentia.webnode.es/200000031-e2181e310b/ia.pdf

Moreira, M. A. (2004). Investigación básica en educación en ciencias: Una visión personal. Revista chilena de educación científica, 3(1), 10-17. Recuperado de https://www. if.ufrgs.br/ moreira/Investigacion.pdf

Moreira, M. A. y Greca, I. M. (2003). Cambio Concetual: Análisis crítico y propuestas a la luz de la teoria del aprendizaje significativo. Ciência e Educação, Bauru, 9, 301-315. Recuperado de http://www.scielo.br/pdf/ciedu/v9n2/10.pdf

Moreira, M. A., Caballero, M. C. y Rodríguez, M. L. (Org.) (1997). Aprendizaje Significativo: Un Concepto Subyacente. En, M. L. Rodríguez, Actas del Encuentro Internacional sobre el Aprendizaje Significativo (pp. 19-244). Burgos, España. Recuperado de https:// www.if.ufrgs.br/ moreira/apsigsubesp.pdf

Moreno, A. M. (2016). Diseño de una Estrategia Didáctica para el Aprendizaje Significativo de los Principios de las Ciencias Naturales Física en el Grado 10, mediante el Diseño y Construcción de un Vehículo de Tracción Humana VTH. [Trabajo grado]. Universidad Cooperativa de Colombia, Bogotá, D.C., Colombia. Recuperado de http://repository.ucc. edu.co:8082/handle/20.500.12494/534

Naranjo, M. L. (2009). Motivación: perspectivas teóricas y algunas consideraciones de su importancia en el ámbito educativo. Revista Educación, 33(2), 153-170. Disponible en https://revistas.ucr.ac.cr/index.php/educacion/article/view/510

Naupas, H., Valdivia, M., Palacios, J. y Romero, H. (2016). Metodología de la investigación cuantitativa, cualitativa y redacción de tesis. (5 ed.). Bogotá, D.C.: Ediciones de la U.

Oliva, J. M. (1999). Algunas reflexiones sobre las concepciones alternativas y el cambio conceptual. Ensenanza de las Ciencias, 17(1), 93-197. Recuperado de https://www.raco. cat/index.php/Ensenanza/article/view/21563

Otero, J. L. (2014). Breve manual para elaborar Secuencia Didáctica. [Online]. Recuperado de https://docplayer.es/21918756-Breve-manual-para-elaborar-secuencia-didactica. html

Pozo, J. I. (1987). La Historia se Repite: Las Concepciones Espontaneas Sobre el Movimiento y la Gravedad. Infancia y Aprendizaje, 10(38), 69-87. Recuperado de https:// dialnet.unirioja.es/servlet/articulo?codigo $=662336$ 
Reiss, M., Millar, R. \& Osborne, J. (1999). Beyond 2000: Science education for the future. Journal of Biological Education, 33(2), 68-70. https://doi.org/10.1080/00219266.1999. 9655644

República de Colombia. MEN. (2006). Estándares Básicos de Competencias. [Online]. Recuperado de https://www.mineducacion.gov.co/1759/articles-81033_archivo_pdf.pdf

Romero, F. (2009). Aprendizaje significativo y constructivismo. Temas para la educación, revista digital para profesionales de la enseñanza, (3), 1-8. Recuperado de https:// www.feandalucia.ccoo.es/docu/p5sd4981.pdf

Ryan, R. \& Deci, E. (2000). Intrinsic and Extrinsic Motivations: Classic Definitions and New Directions. Conteporary Educational Psychology, 25(1), 54-67. https://doi.org/10.1006/ ceps.1999.1020

Sabariego, J. M. y Manzanares, M. (2006). Alfabetización científica. Ponencia presentada en el I Congreso Iberoamericano de Ciencia, Tecnología e Innovación, CTS+I, México, D.F.

Sandín, M. P. (2003). Tradiciones en la investigación cualitativa. En, Investigación cualitativa en educación. Fundamentos y tradiciones (pp. 1-70). México, D.F.: McGraw-Hill Interamericana.

Silva, R. (2011). La enseñanza de la física mediante un aprendzaje significativo y cooperativo en Blended Learnig. [Tesis doctoral]. Universidad de Burgos, España. Recuperado de https://core.ac.uk/download/pdf/61545478.pdf

Tuan, H. L., Chin, C. C. \& Shieh, S. H. (2005). The development of a questionnaire to measure students' motivation towards science learning. International Journal of Science Education, 27(6), 639-654. https://doi.org/10.1080/0950069042000323737

UNESCO. (2003). Informe final del encuentro sobre educación científica. [Online]. Recuperado de https://studylib.es/doc/394815/informe-final-del-encuentro-sobre-eduacióncientífica

María Cristina Murillo Durán es Química de la Universidad Industrial de SantanderUIS (Colombia). Docente de Química y en ciencias de la educación a nivel de Maestría. Actualmente docente de química en la Escuela de Química de la UIS y docencia (presencial, distancia, virtual) en la UIS, UNAB, UExternado de Colombia. Asesora de proyectos educativos (directora de tesis), asesora de proyectos E-Learning (directora de tesis), diseño de Cursos Virtuales (UIS, UNAB), OVAS. https://orcid.org/0000-0001-6223-5452

Elvira Tirado Santamaría es Ingeniera Química egresada de la Universidad Industrial de Santander (Colombia), dedicada a las ciencias de la educación. Actualmente se desempeña como docente de química en el Instituto Técnico Agropecuario de Hato (Santander, Colombia), una institución educativa de carácter técnico ubicada en una zona rural. https:// orcid.org/0000-0001-6357-5475 